\title{
Density and temperature dependence of carrier dynamics in self-organized InGaAs quantum dots
}

\author{
T B Norris ${ }^{1}, K_{\text {Kim }}{ }^{1}$, J Urayama $^{1}$, Z K Wu ${ }^{1}$, J Singh $^{2}$ and \\ P K Bhattacharya ${ }^{2}$ \\ ${ }^{1}$ Center for Ultrafast Optical Science and Department of Electrical Engineering and \\ Computer Science, University of Michigan, 2200 Bonisteel Blvd, Ann Arbor, \\ MI 48109-2099, USA \\ ${ }^{2}$ Solid State Electronics Laboratory, Department of Electrical Engineering and Computer \\ Science, University of Michigan, Ann Arbor, MI 48109-2122, USA
}

Received 13 April 2005

Published 17 June 2005

Online at stacks.iop.org/JPhysD/38/2077

\begin{abstract}
We have used two- and three-pulse femtosecond differential transmission spectroscopy to study the dependence of quantum dot carrier dynamics on temperature. At low temperatures and densities, the rates for relaxation between the quantum dot confined states and for capture from the barrier region into the various dot levels could be directly determined. For electron-hole pairs generated directly in the quantum dot excited state, relaxation is dominated by electron-hole scattering, and occurs on a $5 \mathrm{ps}$ time scale. Capture times from the barrier into the quantum dot are of the order of $2 \mathrm{ps}$ (into the excited state) and $10 \mathrm{ps}$ (into the ground state). The phonon bottleneck was clearly observed in low-density capture experiments, and the conditions for its observation (namely, the suppression of electron-hole scattering for nongeminately captured electrons) were determined. As temperature increases beyond about $100 \mathrm{~K}$, the dynamics become dominated by the re-emission of carriers from the lower dot levels, due to the large density of states in the wetting layer and barrier region. Measurements of the gain dynamics show fast (130 fs) gain recovery due to intradot carrier-carrier scattering, and picosecond-scale capture. Direct measurement of the transparency density versus temperature shows the dramatic effect of carrier re-emission for the quantum dots on thermally activated scattering. The carrier dynamics at elevated temperature are thus strongly dominated by the high density of the high energy continuum states relative to the dot confined levels. Deleterious hot carrier effects can be suppressed in quantum dot lasers by resonant tunnelling injection.
\end{abstract}

\section{Introduction}

Recent years have seen major advances in the development of high performance quantum dot devices, and the application of bandgap engineering to mid-infrared coherent sources and detectors. Although it has been predicted for many years that quantum dots would enable improved performance for optoelectronics, and even the possibility of completely novel devices, it is only in the past few years that the promise of quantum dots has begun to be realized. For example, the recently developed tunnelling-injection quantum dot laser (using self-organized quantum dots in the InGaAs system) significantly exceeds quantum well lasers in a number of important ways (including threshold current, temperature dependence and linewidth enhancement factor) [1]. Quantum dots are also showing promise for a number of applications in quantum information, including deterministic single-photon sources for secure quantum communications [2], and as quantum logic gates for quantum computation [3]. 
The advances in infrared optoelectronics using semiconductor nanostructures have been equally spectacular. Following the development of quantum-well infrared photodetectors in the late 1980s and early 90s, the use of quantum dots in the active region of mid-infrared detectors has enabled trivial normal-incidence operation and higher temperature operation [4]. The quantum cascade laser is of course the best known of the new devices, as it constitutes an entirely new concept in semiconductor laser operation, and has resulted in devices covering the mid-infrared to the terahertz, with many real-world applications now in place.

Critical to the development and understanding of all these devices has been an understanding of the dynamics of the electrons and holes in the active region. Specifically, the understanding of carrier-phonon relaxation (including the phonon bottleneck in quantum dots), carrier-carrier scattering and tunnelling, are central to these devices. For example, in a quantum-dot interband diode laser, electrons and holes injected from the contact regions must be captured into the dots and relax to the ground state, and these energy relaxation processes play a role in determining the ultimate modulation bandwidth attainable with these lasers. In addition, thermally activated carrier escape from the confined dot states, via phonon scattering or carrier-carrier processes, plays a key part in determining threshold current and characteristic temperature $T_{\mathrm{o}}$. Similar arguments apply to quantum-dot infrared detectors (QDIPs). Capture of optically excited electrons back into the dots reduces the efficiency, and thermally activated excitation out of the dots gives rise to dark current in these detectors.

In semiconductor quantum dots, both carrier-phonon and carrier-carrier processes become more active with temperature. When the temperature is raised, the phonon population $n\left(\omega_{0}\right)$ increases. As a result, in the case of acoustic phonons, the single-phonon scattering rate increases proportionally with temperature, and for longitudinal optical phonons the emission and absorption rates increase linearly with $n\left(\omega_{\mathrm{o}}\right)+1$ and $n\left(\omega_{\mathrm{o}}\right)$, respectively. The increase in phonon population also contributes to higher probability for multiphonon scattering, which can be an important mechanism for carrier capture and escape between the confined dot states and the barrier continuum. Furthermore, the carrier-carrier scattering rate becomes faster as the kinetic energy increases with temperature. In the high-density regime, higher-order Auger processes become more likely. The interplay of these effects determines the overall relaxation and excitation processes in devices.

These temperature-dependent processes give rise to thermal re-remission of carriers from the confined dot states into the wetting layer (WL) and barrier continuum and mediate nonradiative recombination (NR), contributing to the temperature-sensitive operation of devices. For example, in multi-stacked quantum dot lasers at room temperature, electroluminescence measurements show a broad asymmetric spectral distribution of carriers among the dot levels due to thermal excitation; this can be strong enough to prevent laser operation in the ground state [5]. Thermal re-emission is also blamed for low photoluminescence (PL) efficiency, a corresponding increase in threshold current and low characteristic temperature above $200 \mathrm{~K}$ [5-7]. Various attempts, such as engineering of the intersubband level spacing, were made to control temperature-sensitive operation, but experiments showed a decrease in characteristic temperature to about $90 \mathrm{~K}$ in the range of 300-325 K [6]. Laser modulation rates were also affected as the modulation bandwidth decreased from greater than $20 \mathrm{GHz}$ at $80 \mathrm{~K}$ to about $5 \mathrm{GHz}$ at $300 \mathrm{~K}$ [8]. NR contributes to the loss of carriers in the dots and degrades device performance at elevated temperature, as reported by many groups [7-10]. Thermal excitation out of the confined dot states is now understood to be a dominant effect in quantum dot structures due to the high density of states in the WL and barrier. The understanding of the role of thermal excitation has led to the development of the quantum-dot tunnellinginjection laser $[1,11]$; in these devices, the thermal excitation problem is overcome by coupling the lasing quantum dot ground state to a quantum well reservoir via resonant phononassisted tunnelling.

Although optoelectronic devices based on quantum dots show performance approaching ideal at low temperature and density, the real-world benefits expected from discrete strongly confined electronic states must withstand the test of higher temperatures and densities. Quantum dot lasers based on traditional carrier injection and capture indeed show degraded performance due to thermally activated processes at room temperature, and thermal activation of electrons giving rise to dark current of course limits the ultimate temperature at which QDIPs can be operated. In order to determine the physical processes underlying device performance, we have applied the technique of femtosecond differential transmission (DT) spectroscopy to directly time-resolve these processes in selforganized InGaAs quantum dots. These studies have enabled the first clear observation of the phonon bottleneck [12], the first observations of electron-hole scattering [13], vertical tunnel coupling [14], gain spectral hole burning and carrier capture into quantum dots in the gain as well as absorption regimes [15], and recently the first time-resolved experiment on tunnelling from quantum wells into quantum dots [11].

In this paper, we review our work on the application of ultrafast DT spectroscopy to directly study the density and temperature dependences of the carrier dynamics. Following a description of the experimental method, we first summarize the dynamics at low density and cryogenic temperature, then consider the effects of elevated temperature. The observed DT signals are interpreted with the aid of a Monte Carlo model for the statistical behaviour of the dynamics. We then describe the dynamics at high density (gain regime) and at low and elevated temperatures.

\section{Experiment and sample}

The sample used for the data shown here was an undoped heterostructure with four layers of $\mathrm{In}_{0.4} \mathrm{Ga}_{0.6} \mathrm{As}$ quantum dots grown by molecular beam epitaxy. The four layers were sandwiched between two $0.1 \mu \mathrm{m}$ thick GaAs layers ('barrier region') and two outer $0.5 \mu \mathrm{m} \mathrm{Al} \mathrm{Al}_{0.3} \mathrm{Ga}_{0.7} \mathrm{As}$ carrier confinement layers, and each dot layer was separated by a $2.5 \mathrm{~nm}$ GaAs barrier layer. The $\mathrm{In}_{0.4} \mathrm{Ga}_{0.6}$ As dot layers were grown at $520^{\circ} \mathrm{C}$ while the rest of the sample was grown at $620^{\circ} \mathrm{C}$. Cross-sectional transmission electron microscopy showed near-pyramidal dots with a base dimension of $14 \mathrm{~nm}$ and a height of $7 \mathrm{~nm}$. Atomic force microscopy showed an 
areal dot density of about $5 \times 10^{10} \mathrm{~cm}^{-2}$ per layer. For DT experiments, the GaAs substrate was removed via selective wet etching to eliminate any possible substrate contribution to the DT signal. Additional discussion of the characterization of similar dot samples is given in $[8,16]$. Experiments on other samples (including InAs dots on GaAs) showed similar results to those reported here, with only small changes to the rate constants for the various scattering processes observed.

Carrier-density-dependent PL data on this sample support the existence of quantum confined dot states and show that the $n=2$ excited state interband transition is centred at $920 \mathrm{~nm}(1.35 \mathrm{eV})$ while the ground state transition $(n=1)$ is centred near $980 \mathrm{~nm}(1.27 \mathrm{eV})$ [13]. This is in reasonable agreement with an eight-band $\mathrm{k} \cdot \mathrm{p}$ band structure calculation, which showed that the interband optical transition matrix element is significant only for those transitions between electron and hole states of the same principal quantum number [14]. The inhomogeneous broadening observed in the PL spectrum is due to dot-size fluctuations with some contribution from miniband formation due to electronic coupling between vertically stacked quantum dots. The PL and DT data and the band structure calculation indicate that the electronic $n=2$ level is about $50 \mathrm{meV}$ below the GaAs conduction band edge, and the spacing between $n=2$ and $n=1$ is about $60 \mathrm{meV}$.

DT measurements were performed over a range of $10-290 \mathrm{~K}$ with an optical pump-probe set-up. A $100 \mathrm{fs}$ $250 \mathrm{kHz}$ amplified $\mathrm{Ti}$ : sapphire laser at $800 \mathrm{~nm}$ was used to generate single-filament white-light continuum sources from which pump and probe pulses were spectrally selected with $10 \mathrm{~nm}$ band-pass interference filters. For three-pulse experiments, a portion of the $800 \mathrm{~nm}$ pulse from the amplifier was split off and used to generate carriers in the sample typically $14 \mathrm{ps}$ before the arrival of the pump pulse. The pump was tuned to generate carriers (or induce stimulated emission) in the ground or excited states of the dots, or in the GaAs barrier continuum states. In degenerate resonant-excitation experiments, the pump and probe were cross-polarized to reduce scattered pump light and a piezo-driven mirror shaker was used to eliminate interference effects between the pump and probe near zero delay. For DT spectral scans, the probe consisted of the dispersion-compensated near-infrared band between 820 and $1050 \mathrm{~nm}$ selected from the white light with a long-pass filter. A monochromator resolved the DT signal to $1 \mathrm{~nm}$ and we detected the probe signal with a lock-in amplifier referenced to the $2 \mathrm{kHz}$ mechanically chopped pump.

When pump-induced carrier population changes are small, the DT signal measures the change in the sum of the electron and hole occupation probabilities of the levels that are in resonance with the pump spectrum. As the probe is delayed with respect to the pump, the dot level population dynamics are time-resolved directly. (When pump-induced population changes are large, i.e. greater than one electron-hole pair per dot, then level shifts induced by many-body Coulomb interactions are observed [17]. For example, the presence of $\geqslant 1$ electron-hole pairs per dot in the $n=1$ state induces a shift of the $n=2$ transition to $930 \mathrm{~nm}$ due to biexcitonic interaction; these effects will be discussed elsewhere as we are primarily concerned in this paper with population dynamics.)

The sample was mounted on a sapphire substrate in a He-flow cryostat with feedback-heater controller for variabletemperature measurements. As the temperature was varied, we noted the spectral redshift of the dot transition energies in both PL and DT and adjusted the probe wavelength accordingly. In our sample, this shift of the confined dot levels amounted to about $20 \mathrm{meV}$ over the temperature range investigated, and the DT time scans did not show significantly different behaviour between shifted and non-shifted probes, especially at higher temperatures (due to the large inhomogeneous broadening present in self-organized dots).

\section{Low density and temperature}

The benefits of quantum dots for optoelectronics arise from the discrete density of the confined states, and are most apparent under strong confinement. The discrete nature of the density of states also strongly modifies intersubband relaxation rates. If the confinement is sufficiently strong that the level spacing exceeds the optical phonon energy, then relaxation due to electron-phonon scattering should be greatly suppressed, an effect known as the phonon bottleneck $[18,19]$. A long excitedstate lifetime would be extremely beneficial for the operation of infrared detectors, as that increases the collection efficiency of photoexcited electrons. On the other hand, a phonon bottleneck would be deleterious for interband quantum dot lasers, since fast intersubband relaxation is desired for high modulation bandwidth.

Optical investigation of the phonon bottleneck requires that experiments be performed at low carrier densities to avoid electron-electron scattering and Pauli exclusion due to state filling, and at low temperature to suppress thermal emission into the continuum, stimulated phonon scattering and direct relaxation of carriers in the high energy tail of the continuum. DT experiments were performed because they directly probe the sum of the electron and hole distributions in each level in the dots; this is in contrast to PL measurements, which measure the product of the electron and hole occupations and, therefore, requires both species to be present in the dot levels with the same quantum number $n$, and therefore is not a general measure of the separate electron and hole dynamics.

The results of our low-density, low-temperature DT experiments have been previously reported in $[12,13]$. When the pump pulse was tuned to resonantly generate electronhole pairs in the $n=2$ level, and the probe was tuned to monitor either the $n=2$ or $n=1$ populations, we found that carriers rapidly relaxed from the $n=2$ to $n=1$ level, contrary to expectations from the phonon bottleneck theory. In particular, the hole relaxation time was found to be $0.6 \mathrm{ps}$ and the electron relaxation time constant was $5.2 \mathrm{ps}$. The fast relaxation of the holes is due to the small quantum dot level spacing in the valence band, enabling rapid phononmediated relaxation. The broadening of the hole states due to the coupling with the phonons relaxes the energy conservation requirement in electron-hole scattering, thus opening up a rapid channel for electronic energy relaxation. That is, an electron can relax from $n=2$ to $n=1$ by scattering a hole to higher energy; the time constants observed were found to be consistent with a numerical model for the scattering. Thus, electron-hole scattering circumvents the phonon bottleneck in a resonantly pumped quantum dot.

The situation becomes more interesting when electronhole pairs are photogenerated in the barrier continuum states 
(pump wavelength $800 \mathrm{~nm}$ ). When free carriers are generated in the barrier region, they quickly thermalize and cool to the GaAs band edge, followed by capture into the dots. At low density, defined here as less than one photo-injected electronhole pair per quantum dot, the capture may take either of two forms, as illustrated in figure 1. The electron and hole may be captured geminately, i.e. the electron and hole are captured into the same dot, or they may be captured nongeminately, i.e. into different dots. There is a higher probability for geminate capture, since, for example, if a hole is first captured, the probability for capture of an electron increases due to Coulomb attraction. Nevertheless, a significant probability exists for generation of nongeminately captured electrons and holes. For this population, the electron-hole scattering mechanism for relaxation discussed above is turned off; electrons captured into the dot $n=2$ level will, therefore, be able to relax only by multiphonon emission, and thus will be bottlenecked in the excited state. Geminately captured electrons, on the other hand, will relax rapidly to the ground state via electron-hole scattering.

The results of a carrier capture experiment at low temperature $(40 \mathrm{~K})$ and density $(<1$ photo-injected pair per dot) are shown in figure 2 . The average population of the dot $n=2$ states is given by DT of a probe pulse tuned in resonance with the $n=2$ transition. The rise time of the signal corresponds to the capture from the barrier into

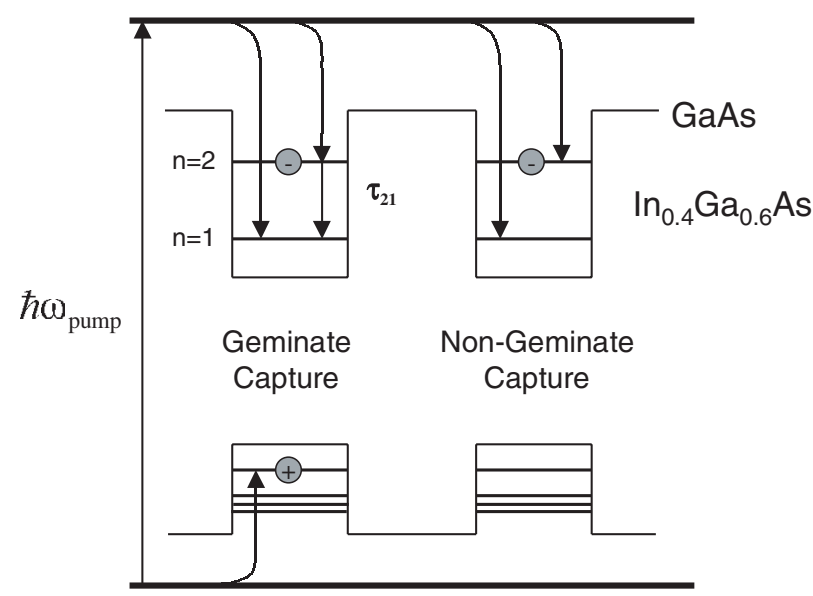

Figure 1. Model of carrier capture processes in quantum dots, showing geminate capture (electron and hole captured into same quantum dot) versus nongeminate capture (into different dots). the $n=2$ level, and the fast decay corresponds to the relaxation of geminately captured electrons. The slow decay component arises from those electrons that are nongeminately captured and thus remain in the excited state due to the phonon bottleneck. It is important to note that this population can only be seen in DT experiments; PL experiments are insensitive to nongeminately captured carriers since the electron and hole must both be present in the dot for recombination to occur. A stringent test of this interpretation of the data is given by the density dependence. If much less than one electron-hole pair per dot is injected into the sample by the pump pulse, then the probability for nongeminate capture is reasonably high. As we have shown in [20], when the pump fluence is increased such that the injected pair density approaches one pair per dot, the probability of nongeminate capture goes down. Thus, the relative amplitude of the slow relaxation component in the $n=2$ DT signal should decrease relative to the amplitude of the fast component as the pump fluence increases. This is exactly the opposite of what is expected if the slow component arises from other mechanisms, e.g. state filling and Pauli exclusion. The experiments indeed showed this behaviour, and were confirmed with a Monte Carlo model (discussed further below), verifying the presence of a phonon bottleneck for nongeminately captured electrons at low density and temperature.

The consequences of these measurements for devices are quite fortuitous. Because electron-hole scattering enables rapid relaxation into the ground state, quantum dot lasers operating on interband transitions are not limited in modulation bandwidth by a phonon bottleneck (we shall see below that the limitations arise from carrier evaporation from the dot confined states into the WL and barrier). On the other hand, unipolar devices based on intersubband transitions, such as QDIPs, have only electrons present in the active region, so electron-hole scattering is not present, and the upper state lifetimes are long, as desired.

\section{Low density: temperature dependence}

At low temperature and density, electrons and holes are captured into the dot confined levels and relax towards the ground state. As the temperature is increased, carrier evaporation or re-emission from the confined levels is possible due to thermally activated phonon absorption, and this effect can be strong at elevated temperatures due to the large

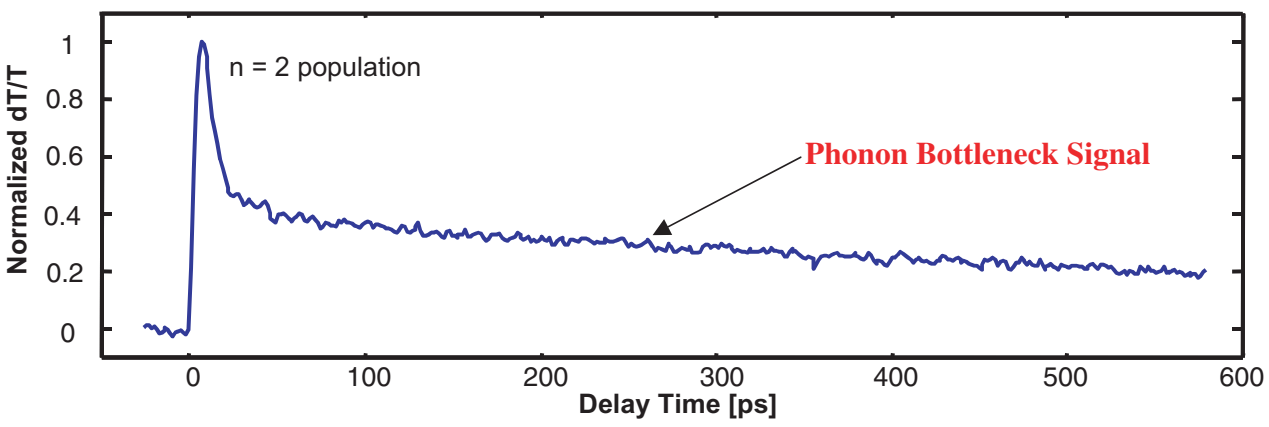

Figure 2. Dynamics of DT signal for probe tuned to quantum dot excited state $(n=2)$, where the pump generates electron-hole pairs in the barrier continuum. The fast decay is due to relaxation of geminately captured electrons via electron-hole scattering, and the slow component is from nongeminately captured electrons trapped in the excited state by the phonon bottleneck. 
density of states in the WL and barrier. Carrier redistribution within the dot levels with increased temperature is evident in time-integrated PL data taken over the temperature range of $12-290 \mathrm{~K}$ (figure 3). This set of data was obtained using $80 \mathrm{fs}$ pulses centred at $800 \mathrm{~nm}$ from a $76 \mathrm{MHz} \mathrm{Ti}$ : sapphire oscillator. The carrier density generated in the barrier region was high enough such that the PL showed both the inhomogeneously broadened $n=1(980 \mathrm{~nm})$ and the $n=2$ $(920 \mathrm{~nm})$ transitions. As the temperature was raised, there was a significant drop in the integrated PL above $80 \mathrm{~K}$ and a slight redshift of the spectrum at the highest temperatures. Both these features have been observed by many groups and the decreased PL efficiency is attributed to thermal re-emission and NR.

To obtain the dynamical behaviour as a function of temperature, we performed DT measurements with resonant excitation into $n=2$ at an average carrier density of less than one electron-hole pair per dot. This low-density regime allows us to consider relaxation without the complications of high-order carrier-carrier scattering processes. The $n=2$

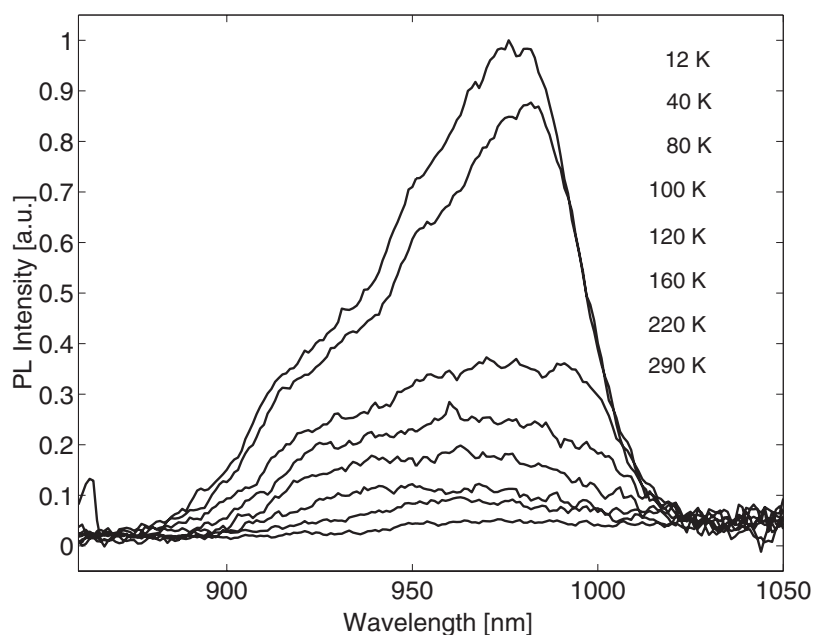

Figure 3. Time-integrated PL measured at 12, 40, 80, 100, 120, 160,220 and $290 \mathrm{~K}$.

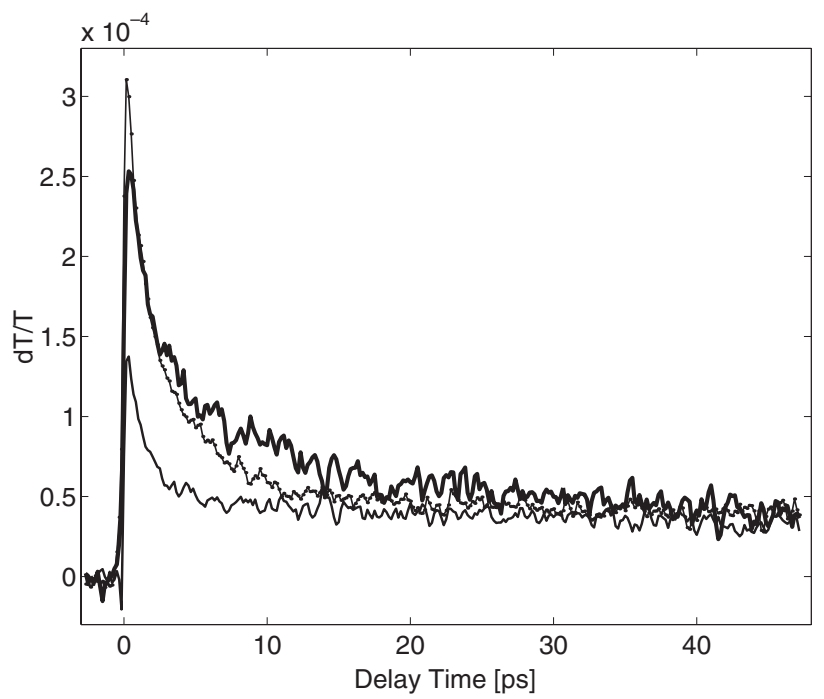

Figure 4. Resonant-excitation DT time scan of the $n=2$ population at $20 \mathrm{~K}$ (dark solid), $80 \mathrm{~K}$ (dotted) and $220 \mathrm{~K}$ (light solid line).
DT data for 20,80 and $220 \mathrm{~K}$ are plotted in figure 4 . The main decay feature at early times corresponds to out-scattering of the $n=2$ population, which was directly generated in the dots. With increased temperature, this decay appears to become faster. We performed a simple three-exponential fit to the data accounting for the very fast sub-picosecond hole decay, the electronic intersubband decay $\tau_{21}$, and the long decay which we attribute to a nongeminate population of carriers induced by electron-hole scattering (the electron-hole scattering process can eject carriers from the dot, which can then be re-captured into another dot forming the nongeminate configuration with nonzero probability) [12]. In table 1 , we record the electronic intersubband relaxation times, $\tau_{21}$, versus temperature.

From 15 to $120 \mathrm{~K}$, the intersubband decay time becomes faster with a minimum near $120 \mathrm{~K}$. This intersubband relaxation has been attributed to electron-hole scattering [13], and these numbers suggest that this mechanism increases in rate with temperature. This trend may result when a hot hole is re-emitted into the high-DOS WL in the electron-hole scattering process. At higher temperatures, the $n=2$ DT signal decreases in magnitude indicating that re-emission (and perhaps NR) dominates the dynamics; we cannot reliably extract an intersubband scattering time at the higher temperatures because of the complications introduced by these processes.

Ground state $(n=1)$ DT time scans are presented in figure 5. Here, the pump was resonant with the excited-state transition $(n=2)$, and we monitored the rate at which the

Table 1. Intersubband relaxation time $\tau_{21}$ and capture time from the barrier into the quantum dots $\tau_{\text {capture }}$ as determined from rate equation fits to the DT dynamics as a function of temperature.

\begin{tabular}{rll}
\hline$(\mathrm{K})$ & $\tau_{21}(\mathrm{ps})$ & $\tau_{\text {capture }}(n=1)(\mathrm{ps})$ \\
\hline 15 & 8.0 & 7.8 \\
40 & 7.5 & 9.4 \\
80 & 7.0 & 5.5 \\
100 & 6.5 & 7.2 \\
120 & 2.8 & 5.3 \\
140 & 3.5 & - \\
180 & 4.0 & - \\
\hline
\end{tabular}

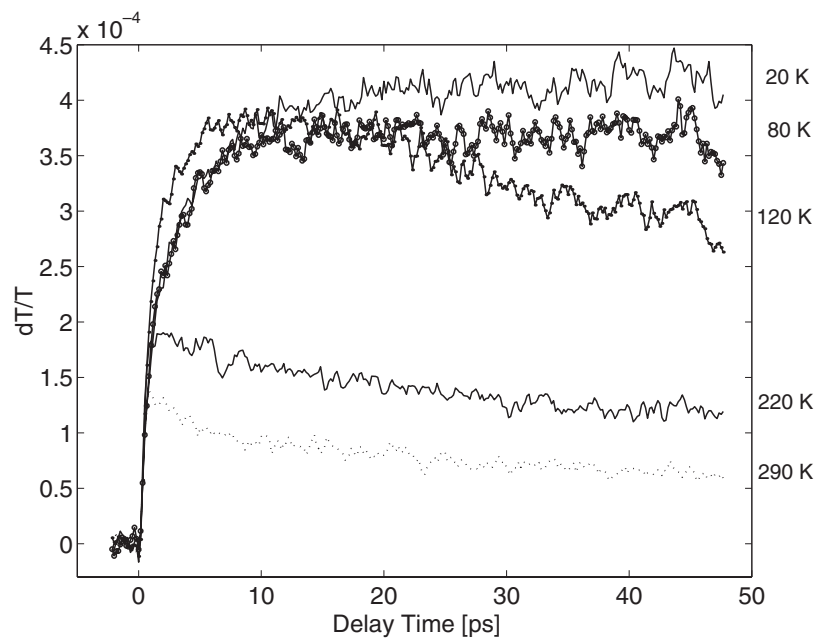

Figure 5. Resonant-excitation DT time scan of the $n=1$ population at $20,80,120,220$ and $290 \mathrm{~K}$. 

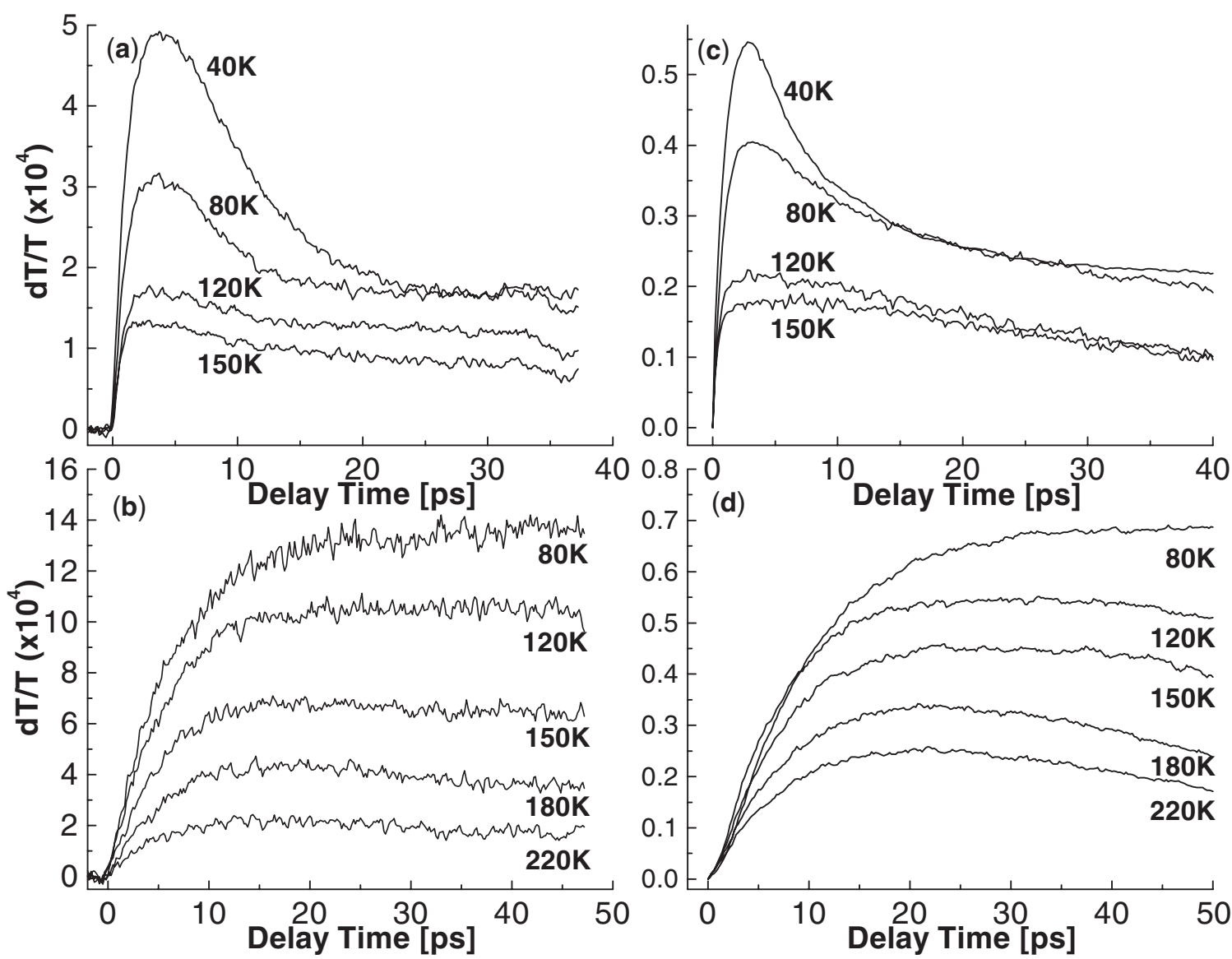

Figure 6. Non-resonant-excitation DT time scans. (a) $n=2$ signal at 40, 80, 120 and $150 \mathrm{~K}$. (b) $n=1$ signal at $80,120,150,180$ and $220 \mathrm{~K}$. Monte Carlo simulation of $(c) n=2$ and $(d) n=1$ population dynamics. The vertical axis in $(c)$ and $(d)$ represent the average number of carriers per dot.

ground state population rises. Similar exponential fits as above were performed on the $n=1$ time scans and are listed in table 1. At low temperatures, the filling rate of the $n=1$ level is comparable to the decay rate of the $n=2$ as reported in [13]. The data in figure 5 show that, beyond $120 \mathrm{~K}$, there is a significant reduction in the signal magnitude due to hole thermalization, carrier re-emission and NR. Accompanying this signal reduction is a faster rise of the $n=1$ population, which could be a result of direct capture of re-emitted carriers from the high-lying states through phonon emission. The effects of re-emission and NR are strong enough at $120 \mathrm{~K}$ that the decay of the ground state occurs very early unlike the radiative decay seen in time-resolved PL measurements at low temperatures. As the temperature is increased further, this trend continues with a significant reduction in the $n=1$ population governed by re-emission and NR. As with the $n=2$ DT data, we cannot draw definitively from these scans a single intersubband relaxation rate at high temperatures because of the influence of these thermally driven processes.

We now consider non-resonant-excitation DT experiments in which a carrier density of less than one electron-hole pair per dot was generated in the GaAs barrier layers with $800 \mathrm{~nm}$ pump pulses. In figure $6(a)$ ), the $n=2 \mathrm{DT}$ time scans measured at $40-150 \mathrm{~K}$ are plotted. Below $80 \mathrm{~K}$, we observed the carrier capture into the excited state of the dot and a subsequent fast and slow relaxation out of the $n=2$ level. The fast component is assigned to relaxation of geminately captured electrons via electron-hole scattering. The slow component is attributed to bottlenecked nongeminately captured carriers. When the temperature increases, the fast component of the $n=2 \mathrm{DT}$ signal decreases more significantly in magnitude than the slow, nongeminate component. This suggests that electronhole scattering plays a role in the re-emission of geminately captured carriers as will be explained below. By $120 \mathrm{~K}$, most of the geminate population disappears. As the temperature increases further beyond $120 \mathrm{~K}$, the overall $n=2$ DT signal diminishes with the decay proceeding with a very long time constant. This trend continues through room temperature (not shown here). The data for the $n=1 \mathrm{DT}$ time scans are given in figure $6(b)$ for the temperature range of $80-220 \mathrm{~K}$. (The data from below $80 \mathrm{~K}$ are omitted because these scans overlap and are very close to the $80 \mathrm{~K}$ DT scan in magnitude and shape.) Here, we see the build up of the ground state population, which again decreases in magnitude at higher temperatures, much like in the resonant-excitation case. As above, in the resonant-excitation case, we attribute the temperaturedependent reduction of the DT signal to thermal re-emission and NR.

The direct observation of the thermal excitation of carriers from the confined dot states to the WL and barrier regions was made through DT time scans of the energy states within $\pm 20 \mathrm{meV}$ of the GaAs band edge. As the temperature 


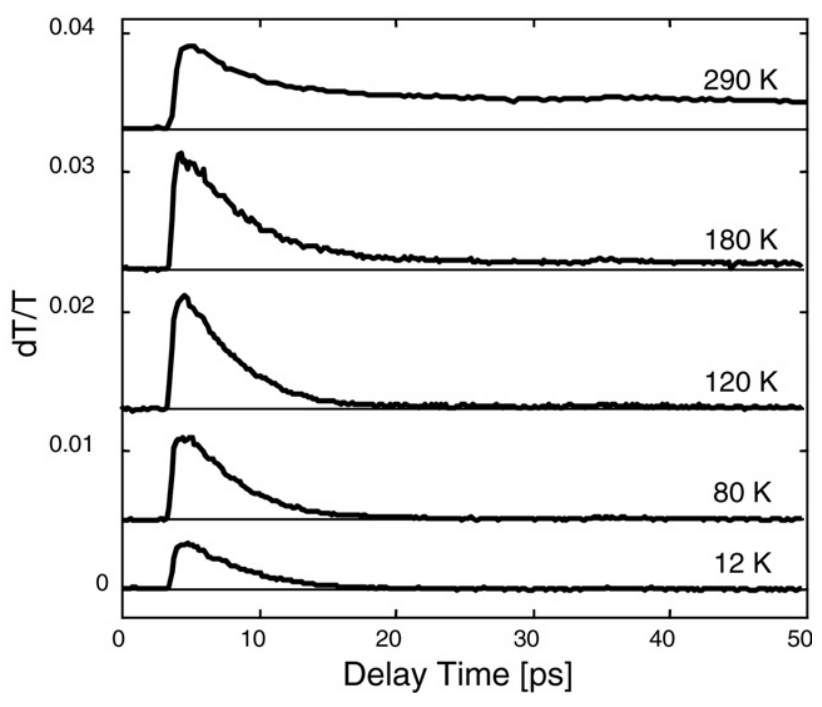

Figure 7. Non-resonant-excitation DT time scan of WL and barrier region ( $\pm 20 \mathrm{meV}$ of $\mathrm{GaAs}$ band edge) measured at $12,80,120,180$ and $290 \mathrm{~K}$.

was varied, this spectral window was adjusted to match the temperature-dependent band edge shift, which was recorded first through DT spectral scans. The DT results show in figure 7 that at low temperatures, the carriers generated in the barrier region all relax down into the lower lying WL and dot levels as indicated by the lack of a signal after the fast initial decay. Around $120 \mathrm{~K}$, a nonzero carrier population begins to emerge in the $\mathrm{WL} / \mathrm{GaAs}$ barrier states. Above $180 \mathrm{~K}$, there is clearly a significant fraction of the carrier population occupying these high-lying states. From spectral scans taken around the band edge, we know that most of the DT signal in the detection spectral window represents carriers that lie in the barrier states, and we know that Pauli blocking is not occurring in the dot levels to produce this barrier signal because the spectral scans show that the overall DT amplitude of the confined dot levels decreases in magnitude at higher temperatures.

We have used an ensemble Monte Carlo simulation to interpret and confirm the consistency of the observed set of temperature-dependent DT signals [20]. The Monte Carlo analysis takes into account the random nature of the scattering processes and is especially appropriate for low-carrier-density experiments in which random geminate and nongeminate capture processes come into play. The model consisted of an ensemble of identical dots, each of which has an energy spectrum obtained from a k $\cdot \mathrm{p}$ bandstructure calculation [21] (see figure 8 for the electronic and hole energy level diagram and the relevant transitions). The DOS of the WL is rather complex and not well characterized. Thus, as an approximation, we include a linearized DOS to account for thermal re-emission channels. The GaAs barrier region is approximated as a quantum well with its 2D DOS. The confined ground and first excited levels have a degeneracy of two and four, respectively, accounting for spin and dot geometry. Both the electron and hole dynamics are included because some of the observed behaviour appears to depend on the geminate and nongeminate nature of the carrier capture. The electron and hole capture, relaxation and re-emission rates within a single

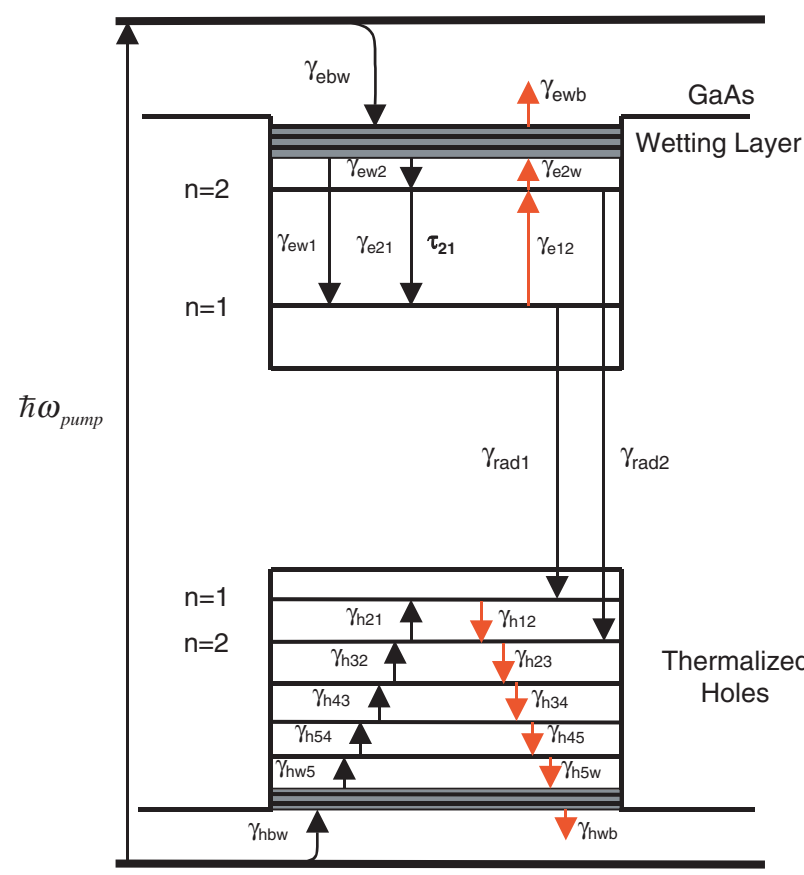

Figure 8. Energy level diagram for the quantum dot structure used in the Monte Carlo simulations.

dot are given in the general form:

$$
\gamma_{\mathrm{e}, \mathrm{h}, \mathrm{i}, \mathrm{f}}=n_{\mathrm{i}} d_{\mathrm{f}}\left(1-\frac{n_{\mathrm{f}}}{d_{\mathrm{f}}}\right)\left(\frac{1}{\tau_{\mathrm{ij}}}\right) \beta .
$$

The indices e and $\mathrm{h}$ refer to the electron and hole rates, and the $i$ and $f$ refer to the initial and final states, the latter with degeneracy $d_{\mathrm{f}}$. The $n_{\mathrm{i}}$ and $n_{\mathrm{f}}$ are the number of occupied states, and $\tau_{\mathrm{ij}}$ is the time constant for the $\mathrm{i}$ to $\mathrm{j}$ transition. $\beta$ is the Boltzmann factor, $\exp \left(-\Delta E_{\mathrm{ij}} / k T\right)$, included for the intradot remission [22,23]. For re-emission between the excited level of the dot and the WL, the above expression was modified by a multiplicative factor of $k T[24,25]$, and the degeneracy $d_{\mathrm{f}}$ was replaced by the number of accessible states in the WL (estimated to be about 100 per dot area). This carrier escape expression assumes that the relaxation and re-emission evolve towards a quasi-Fermi equilibrium and that the quasi-Fermi level is far below the WL band edge. At high temperatures, NR was necessary to account for carrier loss. We included this in the model as a simple decay from the entire system as a function of temperature. Radiative recombination decay times measured in time-resolved PL experiments were also included in the model, although they do not play a significant role in the early dynamics. In producing the simulated DT signals with this model, we used the time constants $\tau_{\mathrm{ij}}$ as adjustable parameters. In an effort to simplify the model, we adjusted only those parameters that captured the main features observed in the DT time scans, noting that most of the parameters are constrained by the resonant-excitation measurements and the WL/barrier data.

Some additional conditions on the scattering processes were included in the model. First, the enhancement of geminate capture due to Coulomb interaction was accounted for by a multiplicative Coulomb enhancement factor (CEF). This factor increases the probability that an electron falls 
into a dot with a hole and was determined from the relative contribution of geminate and nongeminate capture observed in the low temperature DT data [20]. Second, we assumed that the electronic intersubband relaxation is dominated by electronhole scattering and allow the intradot electronic relaxation to proceed only if the dot is occupied by a hole. Third, the injected hole population was kept in a thermal distribution through fast intersubband relaxation and re-excitation, consistent with calculations that show that the holes are well coupled to the lattice through phonons because of the closely spaced hole levels [26].

In figures $6(c)$ and $(d)$ we present simulation results that match the main dynamical features of the non-resonant-pump $n=2$ and $n=1$ DT data. For these results, we used an ensemble of 5000 dots and injected a carrier density of 0.5 electron-hole pairs per dot into the GaAs barrier. The $\mathrm{CEF}$ for the capture process was fixed throughout all the runs such that the nongeminate capture rate was about $20 \%$ of the geminate capture rate. At higher temperatures, the capture times for the electrons (into both the $n=2$ and the $n=1$ levels) and the holes were decreased to account for the faster rise of the $n=2$ and $n=1$ populations. The $n=2(n=1)$ capture rate for the electrons ranged from about $2.5 \mathrm{ps}(40 \mathrm{ps})$ at $40 \mathrm{~K}$ to $2.0 \mathrm{ps}(20 \mathrm{ps})$ at $290 \mathrm{~K}$. For the holes, the capture time into the dots was about $50 \mathrm{ps}$ at $40 \mathrm{~K}$ and $15 \mathrm{ps}$ at $290 \mathrm{~K}$. The increased capture rate with temperature could be attributed to a decreased diffusion time of carriers through the barrier region and the increased resonant capture of thermalized carriers from the WL/barrier region through multiphonon scattering, as was observed at high temperatures in the resonant-excitation case above. Multiphonon-mediated capture would occur when the thermalized carriers reach an energy that is spaced an integral number of available LO phonon energies above the confined dot levels. The temperature-dependent electronic intersubband relaxation time constants obtained from the resonant-excitation DT measurements were used in the model.

With increasing temperature, re-emission and NR begin to dominate the dynamics. In the $n=2$ time scan at $80 \mathrm{~K}$, the geminate component of the carrier population undergoes re-emission at a faster rate than the nongeminate population. This can be attributed to electron-hole scattering in the geminately captured dots in which holes in the high-energy tail of the thermal distribution kick the $n=2$ electrons into the high-DOS WL. We note that this is consistent with the large relative reduction seen between 40 and $80 \mathrm{~K}$ in the PL signal taken at higher carrier density where carrier-carrier scattering is more dominant. For the nongeminate electrons, re-emission is mediated by phonons. In order to account for this, two different re-emission rates were used for the two electron populations. Through $80 \mathrm{~K}$, the magnitude of the $n=1$ population remains relatively constant because the electronic intersubband energy spacing $\left(\Delta E_{21} \approx 60 \mathrm{meV}\right)$ is too large for re-emission to occur from the ground state. Also, the $n=1$ level maintains its population while the $n=2$ population decreases at $80 \mathrm{~K}$ because direct capture of electrons from the $\mathrm{WL} / \mathrm{barrier}$ region occurs to compensate for this reduced intersubband contribution. When the temperature is increased beyond $100 \mathrm{~K}$, the $n=1$ DT signal decreases initially because of thermal redistribution of the holes. Then, both $n=2$ and $n=1$ carriers are re-emitted causing a decrease in the dot DT signal and a build up of the WL/barrier population. Given the degeneracies and spacing of the levels mentioned above, the re-emission time constant for the $n=2$ dot level is of the order of hundreds of picoseconds at lower temperatures. At higher temperatures, this re-emission time is in the tens of picoseconds range. The rate of re-emission was adjusted by monitoring the resulting barrier population as a function of temperature. We found that when the re-emission level was adjusted to fill the barrier region sufficiently, the signal magnitudes in the dot levels were too high. That is, an additional carrier-loss mechanism is required to account for the overall signal reduction. We assigned this carrierloss mechanism to NR, which accounts for low quantum efficiency in high-temperature luminescence experiments. The energy dependence of NR is very complex, but for simplicity we assumed a simple exponential decay from the entire population. This produced a reasonable set of results for the $n=2$ and $n=1$ populations as shown in figures $6(c)$ and $(d)$ as well as that for the barrier region (not shown here). We note that the long term decay of the $n=1 \mathrm{DT}$ signal is governed more by the re-emission and NR rates than by radiative recombination rates as, in the low-carrier-density regime, the nongeminate population does not contribute to radiative recombination which has been reported to become slower with temperature $[9,27,28]$.

The most important result of the DT experiments and the Monte Carlo simulations is that, for temperatures below about $100 \mathrm{~K}$, the effects of carrier capture and the phonon bottleneck are directly manifested in the DT dynamics, but that for higher temperatures, the dynamics are driven largely by the high density of states in the WL or barrier region surrounding the quantum dots. By performing pump-probe spectroscopy, we have been able to directly observe the carriers in these excited states and their dynamics. These results also indicate that the dynamics of optoelectronic devices such as quantum dot lasers and QDIPs are not driven by the intrinsic electron-phonon coupling, but rather by the large density of excited states. Thus, for example, the modulation rate of a laser is not limited by the scattering rate into the ground state, but rather by the thermal re-excitation of carriers out of the optically active low-lying quantum dot states into the WL or barrier. These excited states (many of which are localized due to the large disorder inherent in the system) serve as a parasitic reservoir, which must be modulated by the drive current, but which do not contribute to the lasing (optically coupled) states. The solution to this problem is to utilize resonant tunnelling injection into the lasing ground state of the dots, as presented elsewhere in this issue [1]. In a tunnellinginjection quantum-dot laser, a quantum well is coupled to the quantum dot active region by LO-phonon-assisted resonant tunnelling. Because of the high density of states in the quantum well coupled directly to the lasing states, the effects of carrier evaporation into the WL/barrier are suppressed, and the overall carrier distribution remains quasi-thermal with a high population in the quantum dot ground state even under high injection conditions. Thus, the parasitic effects of the WL/barrier are reduced, enabling substantial improvement of the modulation bandwidth of quantum dot lasers. 


\section{High density: temperature dependence}

The carrier dynamics leading to a phonon bottleneck in the low-temperature, low-carrier-density regime as discussed above do not apply directly in the high-temperature, highcarrier-density regime where room temperature lasers are operated, although the low-density experiments did reveal the important role of electron-hole scattering and the dominant effect of the WL and barrier continuum states on the dynamics at elevated temperature. In order to determine the time constants for carrier capture and relaxation relevant to roomtemperature interband laser operation, it is necessary to perform measurements at high densities corresponding to a population inversion, i.e. to measure the gain dynamics. In the gain regime, carrier-carrier scattering processes dominate.

We have used a three-pulse pump-probe spectroscopic technique to observe the gain dynamics in the same sample discussed above [15]. Carriers were injected by optically pumping the GaAs barrier region with a 'gain pulse' at $800 \mathrm{~nm}$ to establish a population inversion in the quantum dots; the intensity of the gain pulse was adjusted so that the dots could be anywhere in the absorption or gain regime. The gain pulse injected carriers at least 14 ps before the arrival of the 'pump' pulse, allowing the photo-injected carriers to relax completely into the quantum dots and reach a thermal distribution. Tunable pump and probe pulses were generated as before by spectrally filtering two single-filament white-light sources. For spectralhole-burning studies, the probe pulse was spectrally resolved after passing through the sample, thus giving the DT spectrum across the entire spectral range spanning the confined dot states. The three pulses were focused on the quantum dot sample near normal incidence; in this geometry we obtained the intrinsic single-pass gain dynamics in the quantum dots, uncomplicated by any propagation effects or device parasitics that one might have in a $\mathrm{p}-\mathrm{i}-\mathrm{n}$ waveguide structure.

It is important to note that, at low density (below transparency), the effect of the pump pulse is to generate additional carriers in the resonant quantum dot levels, yielding a positive DT probe signal. At high density, when the population is inverted, the pump pulse induces stimulated emission, which reduces the carrier population in the dots, thus producing a negative probe DT signal. If the density is such that the transparency point is at the pump photon energy, then the pump pulse induces no carrier population change, and the probe DT signal is zero; thus, the signature of a population inversion in the system is the change in sign of the probe DT.

The results of the DT gain dynamics when the sample is held at cryogenic temperature were published in [15]. Transient spectral hole burning was observed, enabling us to separate the effects of gain recovery due to intradot carriercarrier scattering and capture into the discrete dot states from the continuum. The gain recovery in the ground state due to carrier-carrier scattering from the $n=2$ state was extremely fast (130 fs in $\mathrm{In}_{0.4} \mathrm{Ga}_{0.6}$ As dots, $180 \mathrm{fs}$ in InAs dots), and the time constant for capture from the barrier region was about $1 \mathrm{ps}$. The presence of a localized spectral hole in both the ground and excited states of the dot confirmed the discrete nature of the DOS.

The transient spectral hole burning results for room temperature are shown in figure 9. The behaviour is similar to

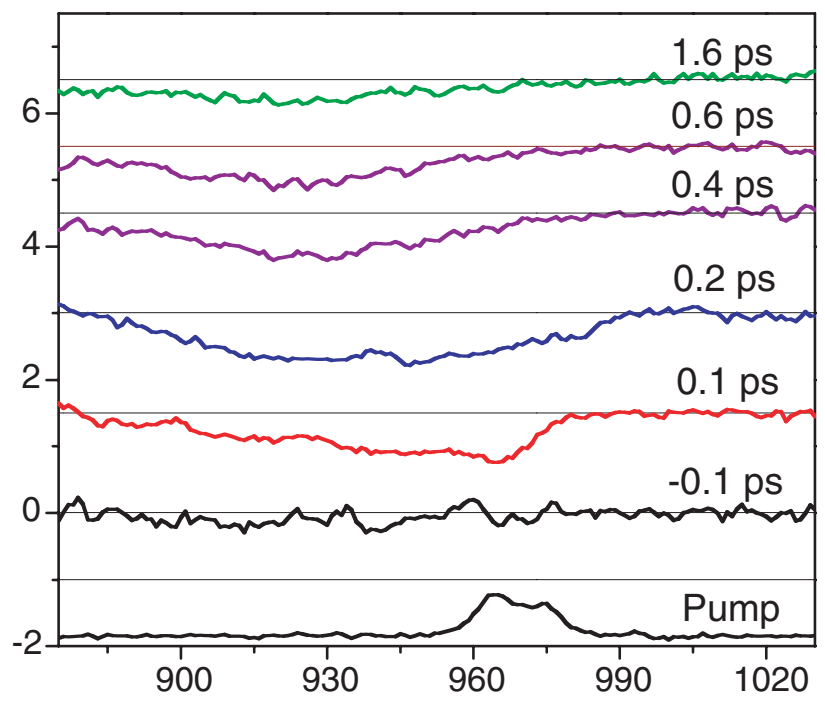

Figure 9. DT spectra at $T=285 \mathrm{~K}$ measured with a 970 pump and a broad band white-light probe for various probe delays (pump pulse at $t=0$, gain pulse at approximately $t=-20 \mathrm{ps}$ ).

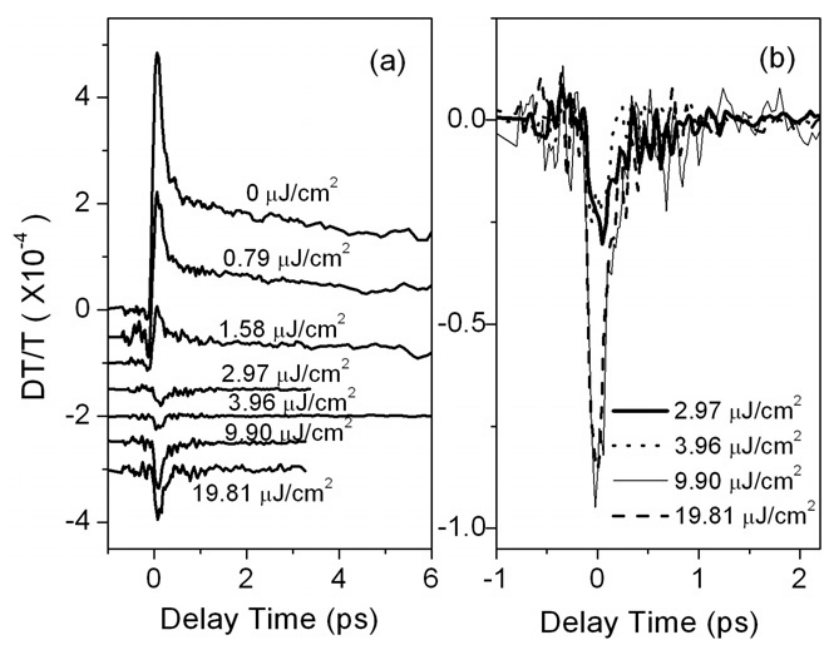

Figure 10. DT time scans at $T=250 \mathrm{~K}$ measured with a $970 \mathrm{~nm}$ pump and probe for different fluences of the gain pulse which injects carriers at $t=-14 \mathrm{ps}$.

that at low temperature; near time zero, the pump pulse burns a hole in the gain spectrum centred at the pump wavelength by depleting the carrier population in the $n=1$ level via stimulated emission. There is a rapid partial recovery of the hole as the $n=1$ population is replenished from the $n=2$ state population due to carrier-carrier scattering. As carriers relax from $n=2$ to $n=1$ on the $100 \mathrm{fs}$ time scale, a satellite spectral hole appears in the gain spectrum at the excited state transition. The excited state spectral hole is significantly broader at room temperature than at low temperature, since thermally activated processes open up scattering channels between quantum dots and thus a more rapid approach to a thermal distribution among the dots. The excited state spectral hole recovers on the singlepicosecond time scale, corresponding to the capture of carriers from the WL and barrier region.

Temporal scans of the DT signal for ground state pump and probe $(970 \mathrm{~nm})$ are shown in figure 10 , at a temperature 


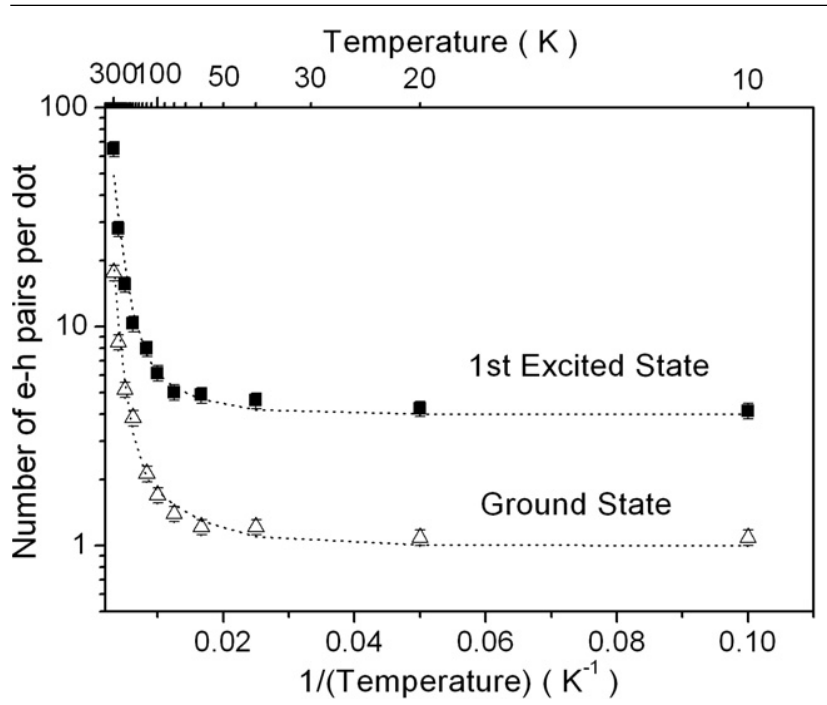

Figure 11. Transparency carrier densities measured with a gain pulse $20 \mathrm{ps}$ before the pump pulse, measured for the ground state $(\boldsymbol{\Delta})$ and excited state (ם)

of $250 \mathrm{~K}$, for various gain pulse fluences (in parentheses). The DT signal is positive (induced transmission) when the gain pulse fluence is zero, and becomes negative when the gain pulse exceeds the transparency fluence of $3 \mu \mathrm{J} \mathrm{cm}^{-2}$ (about 8.5 electron-hole pairs per dot) at $250 \mathrm{~K}$. At higher fluences, the DT signal is negative (induced transmission). The gain recovery dynamics are very fast, and weakly density dependent: for gain fluences of $3 \mu \mathrm{J} \mathrm{cm}^{-2}, 4 \mu \mathrm{J} \mathrm{cm}^{-2}$, $10 \mu \mathrm{J} \mathrm{cm}^{-2}$ and $20 \mu \mathrm{J} \mathrm{cm}^{-2}$, the fast component of the gain recovery determined by rate equation fitting is $207 \pm 21 \mathrm{fs}$, $143 \pm 10 \mathrm{fs}, 147 \pm 13 \mathrm{fs}$ and $134 \pm 6 \mathrm{fs}$, respectively. The amplitudes of the initial pump-induced gain depletion and the fast component of the gain recovery are larger for higher carrier densities.

Because the sign of the DT signal flips as the gain pulse fluence is varied, the three-pulse DT technique provides an accurate and sensitive method to determine the transparency density versus temperature and energy. We have previously reported the results of this technique in [29], but include a brief discussion here for completeness. In those experiments, a gain pulse injected a variable density of carriers into the barrier region, which relaxed towards a thermal distribution (although at the very lowest densities and temperatures, statistical capture into the dots prevents a true thermal distribution from being formed). After a $20 \mathrm{ps}$ delay, a degenerate pump-probe experiment was performed; the transparency condition at the pump-probe transition energy was determined by the gain pulse fluence at which the DT signal flipped sign. The fluences were converted into electron-hole pair densities using published and experimentally verified values of the absorption coefficient and reflectivity. The pump-probe wavelength was $970 \mathrm{~nm}(920 \mathrm{~nm})$ for the ground (excited) state data shown in figure 11. The transparency population for the ground state was found to be 1.1 electron-hole pairs per dot at low temperature, close to the expected value of one. At low temperature, the transparency condition was 4.5 pairs per dot, close to the expected condition of 2 in the $n=1$ state and 2 in the $n=2$ state, confirming that the spatial degeneracy of the excited state is two. As the temperature was increased from 10 to $300 \mathrm{~K}$, the transparency density increased from 1.1 to 18 pairs in the ground state and from 4.5 to 80 pairs per dot in the excited state; the slopes of the transparency curves in figure 11 change dramatically around $100 \mathrm{~K}$.

The experimental results for the transparency versus temperature were compared with a model for the effective density of states in the quantum dot system incorporating the confined dot levels and modelling the barrier as a wide quantum well. The results are also shown in figure 11, and confirm that the dramatic increase in transparency density with temperature above $100 \mathrm{~K}$ arises from thermal excitation into the continuum. Even though the quantum dot ground state is separated from the continuum by many $\mathrm{kT}$ even at room temperature, the DOS of the WL/barrier is so large that the population (which is given by the product of the distribution function and the DOS) in the ground state is small. This is consistent with the dynamics measurements discussed in section 3, and explains the degradation of the threshold current and the modulation bandwith of quantum dot injection lasers with increased temperature.

\section{Conclusions}

Ultrafast optical spectroscopy has been shown to be a powerful tool for determining the dynamics of carrier populations in quantum dot systems. At low density and temperature, the rates of carrier capture and relaxation could be directly determined. For temperatures above $100 \mathrm{~K}$, however, thermally activated processes begin to dominate. The dynamics of optoelectronic devices, such as quantum dot lasers operating above cryogenic temperatures are therefore not driven by the intrinsic electron-phonon coupling, but rather by the large density of excited states. Thus, the modulation rate of a laser is not limited by the scattering rate into the ground state, but rather by the thermal re-excitation of carriers out of the optically active low-lying quantum dot states into the WL or barrier. Similarly, any other quantum dot device will be affected by the thermal excitation into excited states. Based on this knowledge, device design strategies can be implemented to mitigate hot carrier effects; for example, the tunnellinginjection laser effectively suppresses hot carrier parasitic effects and enables temperature-independent operation and high modulation bandwidth.

\section{References}

[1] See Fathpour S, Mi Z and Bhattacharya P 2005 J. Phys. D: Appl. Phys. 382103 (this issue)

[2] Santori C, Pelton M, Solomon G S, Dale Y and Yamamoto Y 2001 Phys. Rev. Lett. 861502

[3] Xiaoqin Li, Yanwen Wu, Steel D, Gammon D, Stievater T H Katzer D S, Park D, Piermarocchi C and Sham L J 2003 Science $\mathbf{3 0 1} 809$

[4] See Chakrabarti S, Stiff-Roberts A D, Su X H, Bhattacharya P, Ariyawansa G and Perera A G U 2005 J. Phys. D: Appl. Phys. 382135 (this issue)

[5] Shoji H, Nakata Y, Mukai K, Sugiyama Y, Sugawara J, Yokoyama N and Ishikawa H 1997 Appl. Phys. Lett. 71193

[6] Schhekin O B, Park G, Huffaker D L and Deppe D G 2000 Appl. Phys. Lett. 77466

[7] Brusaferri L, Sanguinetti S, Grilli E, Guzzi M, Bignazzi A, Bogani F, Carraresi L, Colocci M, Bosacchi A, Frigeri P and Franchi S 1996 Appl. Phys. Lett. 693354 
[8] Bhattacharya P, Kamath K K, Singh J, Klotzkin D, Phillips J, Jiang H, Chervela N, Norris T B, Sosnowski T, Laskar J and Murty M R 1999 IEEE Trans. Electron Devices 46871 and references therein

[9] Zhang L, Boggess T F, Deppe D G, Huffaker D L, Shchekin O B and Cao C 2000 Appl. Phys. Lett. 761222

[10] Xu Z Y, Lu Z D, Yang X P, Yuan Z L, Zheng B Z, Xu J Z, Ge W K, Wang Y, Wang J and Chang L L 1996 Phys. Rev. B 5411528

[11] Bhattacharya P, Ghosh S, Pradhan S, Singh J, Wu Z-K, Kim K and Norris T B 2003 IEEE J. Quantum Electron. 39952

[12] Urayama J, Norris T B, Singh J and Bhattacharya P 2001 Phys. Rev. Lett. 864930

[13] Sosnowski T S, Norris T B, Jiang H, Singh J, Kamath K and Bhattacharya P 1998 Phys. Rev. B 57 R9423

[14] Urayama J, Norris T B, Kochman B, Singh J and Bhattacharya P 2000 Appl. Phys. Lett. 762394

[15] Kim K, Urayama J, Norris T B, Singh J, Phillips J and Bhattacharya P 2002 Appl. Phys. Lett. 81670

[16] Kamath K, Chervela N, Linder K K, Sosnowski T, Jiang H-T, Norris T, Singh J and Bhattacharya P 1997 Appl. Phys. Lett. 71927
[17] Kyoungsik Kim 2004 PhD Thesis University of Michigan

[18] Benisty H, Sotomayor-Torres C M and Weisbuch C 1991 Phys. Rev. B 4410945

[19] Bockelman U and Bastard G 1990 Phys. Rev. B 428947

[20] Urayama J, Norris T B, Jiang H, Singh J and Bhattacharya P 2002 Physica B: Phys. Condens. Matter C 316-317 74

[21] Jiang H and Singh J 1998 Physica E 2614

[22] Yang W, Lowe-Webb R R, Lee H and Sercel P C 1997 Phys. Rev. B 5613314

[23] Sanguinetti S, Henini M, Grassi Alessi M, Capizzi M, Frigeri P and Franchi S 1999 Phys. Rev. B 608276

[24] Romero B, Arias J, Esquivias I and Cada M 2000 Appl. Phys. Lett. 761504

[25] Berg T W, Biscoff S, Magnusdottir I and Mork J 2001 IEEE Photon. Technol. Lett. 13541

[26] Jiang H and Singh J 1997 Phys. Rev. B 564696

[27] Wang G, Fafard S, Leonard D, Bowers J E, Merz J L and Petroff P M 1994 Appl. Phys. Lett. 642815

[28] Yu H, Lycett S, Roberts C and Murray R 1996 Appl. Phys. Lett. 694087

[29] Kim K, Norris T B, Singh J and Bhattacharya P 2003 Appl. Phys. Lett. 821959 